\title{
RATIONAL SINGULARITIES OF HIGHER DIMENSIONAL SCHEMES
}

\author{
ECKART VIEHWEG
}

\begin{abstract}
Two examples of rational singularities of schemes over an algebraically closed field of characteristic zero are given: Singularities occurring as the quotient of a regular scheme by a finite group and singularities of the type $u^{2}-v^{2}-g\left(t_{1}, \ldots, t_{N}\right)$.
\end{abstract}

Unless otherwise explicitly mentioned, we assume that all schemes are irreducible, reduced and of finite type over an algebraically closed field $k$ of characteristic zero and that all points are $k$-rational.

Let $U$ be a scheme, $X$ a regular scheme and $g: X \rightarrow U$ a proper, surjective, birational morphism. We call $g: X \rightarrow U$ a resolution of $U$.

Definition 1. Let $Y$ be a scheme and $y \in Y$. Then $Y$ has a rational singularity at $y$ if there exists a neighbourhood $U$ of $y$ in $Y$, such that for every resolution $g: X \rightarrow U$ we have $g_{*} \theta_{X} \cong \theta_{U}$ and $R^{i} g_{*} \theta_{X}=0$ for $i \neq 0$ (henceforth we write $\mathbf{R}_{*} \theta_{X} \cong \hat{\theta}_{U}$ ).

In this paper we want to discuss two examples of rational singularities needed in $[5, \S 5]$.

RemarKs. (i) Using "flat base change" [1] it is easy to see that the question whether $y \in Y$ is a rational singularity depends only on $\theta_{y, Y}$ ("^" denotes the completion with respect to the maximal ideal).

(ii) If the base field has positive characteristic, one needs additional conditions to define rational singularities [3].

(iii) Every regular point of a scheme is a rational singularity [2].

(iv) In Definition 1 it is sufficient to consider one resolution of $U$.

This last statement follows from (iii) and the Leray spectral sequence. Using the same kind of argument one gets

Lemma 1. Let $h: Y^{\prime} \rightarrow Y$ be a proper, surjective, birational morphism of schemes. Assume that $Y^{\prime}$ has only rational singularities; then $\mathbf{R} h_{*} \theta_{Y^{\prime}} \cong \hat{\theta}_{Y}$, if and only if $Y$ has only rational singularities.

We first consider quotient singularities:

Definition 2. Let $Y$ be a scheme and $y \in Y$. Then $Y$ has a quotient singularity at $y$ if there exist a regular scheme $Y^{\prime}$ and a finite group $G$ acting

Received by the editors June 17, 1976.

AMS (MOS) subject classifications (1970). Primary 14E15, 14B05; Secondary 14E20.

Key words and phrases. Rational singularities, resolution of singularities, higher direct images, quotient singularities, ramified covers, families of stable curves.

(๑) American Mathematical Society 1977 
on $Y^{\prime}$ such that $Y^{\prime} / G$ exists and is isomorphic to some neighbourhood of $y$ in $Y$.

Let $W$ be a regular scheme and $D \subseteq W$ a closed, reduced subscheme of codimension 1 . We say that $D$ has normal crossings if the irreducible components of $D$ are regular and if for every point $w \in W$ regular parameters $x_{1}, \ldots, x_{n}$ exist such that $D$ is defined by $x_{1} \cdot x_{2} \cdot \ldots \cdot x_{r}=0$.

LEMMA 2. Let $W$ be a regular scheme and $f: Y \rightarrow W$ a finite morphism of normal schemes. Assume that the ramification locus $\Delta(Y / W)$ (see [4]) has only normal crossings. Then $Y$ has quotient singularities and $f$ is flat.

Proof. The first statement follows from Abhyankar's lemma: The proof is exactly that used in [4, pp. 32-33] to prove "Satz 4.1". One must simply add "and hence $P_{2}$ is a regular point of $X_{2}$ " after the 18th line of p. 33 .

Since flatness is a local property, we may assume that for some regular scheme $Y^{\prime}$ and for some group $G$ we have $h: Y^{\prime} \rightarrow Y^{\prime} / G \cong Y$. Then $f \cdot h$ is a finite morphism of regular schemes, and hence $(f \cdot h)_{*} \theta_{Y^{\prime}}$ is locally free. Let $\eta: \theta_{Y} \rightarrow h_{*} \theta_{Y^{\prime}}$ be the map "multiplication with $\operatorname{ord}(G)^{-1}$ ", and $\operatorname{Tr}: h_{*} \theta_{Y^{\prime}} \rightarrow$ $\theta_{Y}$ the trace map. Then $\operatorname{Tr} \cdot \eta$ is an isomorphism and, therefore, $f_{*} \theta_{Y}$ a direct summand of $(f \cdot h)_{*} \Theta_{Y^{\prime}}$.

PROPOSITION 1. Every quotient singularity is a rational singularity.

Proof. Assume that for some $n \geqslant 1$ and every scheme $Y$ having quotient singularities we already know:

For every resolution $g: X \rightarrow Y$ we have $R^{i} g_{*} \theta_{X}=0$ for $0<i<n$.

Let $Y=Y^{\prime} / G$ (as in Definition 2) and $g: X \rightarrow Y$ be a resolution. Denote the normalization of $X$ in the function field of $Y^{\prime}$ by $X^{\prime}$ and choose a resolution $h^{\prime}: W^{\prime} \rightarrow X^{\prime}$ of $X^{\prime}$. We denote the natural morphisms by:

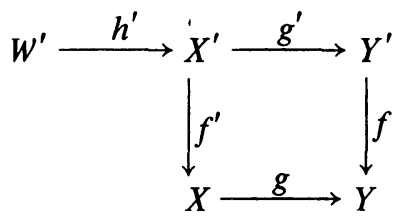

Using "embedded resolution of singularities" [2] we may assume that $\Delta\left(X^{\prime} / X\right)$ has normal crossings. $X^{\prime}$ also has quotient singularities (Lemma 2) and, by assumption, $R^{i} h_{*}^{\prime} \vartheta_{W^{\prime}}=0$ for $0<i<n$. The Leray spectral sequence gives an injection $R^{n} g_{*}^{\prime}\left(h_{*}^{\prime} \theta_{W^{\prime}}\right) \rightarrow R^{n}\left(g^{\prime} \cdot h^{\prime}\right)_{*} \theta_{W^{\prime}}$. Since $g^{\prime} \cdot h^{\prime}$ is a resolution of a regular scheme, $R^{n}\left(g^{\prime} \cdot h^{\prime}\right)_{*} \theta_{W^{\prime}}=0$ and therefore $0=$ $f_{*} R^{n} g_{*}^{\prime} \theta_{X^{\prime}}=R^{n} g_{*}\left(f_{*}^{\prime} \theta_{X^{\prime}}\right)$. Since $\theta_{X}$ is a direct summand of $f_{*}^{\prime} \theta_{X^{\prime}}$ (proof of Lemma 2), we get $R^{n} g_{*}{ }^{\left({ }^{\prime}\right.}{ }_{X}=0$.

The second example is given by an explicit equation:

Proposition 2. Let $Y$ be a scheme and $y \in Y$ such that

$$
\hat{\theta}_{y, Y}=k\left[\left[t_{1}, \ldots, t_{n}, u, v\right]\right] /\left(u^{2}-v^{2}-g\left(t_{1}, \ldots, t_{n}\right)\right),
$$


$0 \neq g\left(t_{1}, \ldots, t_{n}\right) \in k\left[\left[t_{1}, \ldots, t_{n}\right]\right]$. Then we have:

(i) $\hat{\theta}_{y, Y}$ (and hence $\hat{\theta}_{y, Y}$ ) is Cohen-Macaulay and normal.

(ii) $\hat{\mathcal{O}}_{y, Y}$ is flat over $k\left[\left[t_{1}, \ldots, t_{n}\right]\right]$.

(iii) $y$ is a rational singularity of $Y$.

Proof. $\hat{\theta}_{y, Y}$ is a complete intersection and, hence, Cohen-Macaulay. The normality follows from Serre's criterion.

(ii) is true since the induced morphism of schemes is equidimensional. We may assume that

$$
Y=\operatorname{Spec}\left(k\left[\left[t_{1}, \ldots, t_{n}\right]\right][u, v] /\left(u^{2}-v^{2}-g\left(t_{1}, \ldots, t_{n}\right)\right)\right)
$$

and $W=\operatorname{Spec}\left(k\left[\left[t_{1}, \ldots, t_{n}\right]\right]\right)$. Let $D$ be the subscheme of $W$ defined by $g\left(t_{1}, \ldots, t_{n}\right)=0$. Using "embedded resolution of singularities" [2], "flat base change" [1] and Lemma 1, we may assume that $D_{\text {red }}$ has normal crossings. After choosing another system of regular parameters in $W$, we get $g\left(t_{1}, \ldots, t_{n}\right)=t_{1}^{\nu_{1}} \cdot t_{2}^{\nu_{2}} \cdot \ldots \cdot t_{n}^{\nu_{n}}, \nu_{i} \in \mathbf{N}$. Although singularities of this type are known to be rational [3], we prove it directly:

Step 1. Blow up ideals of the form $\left\langle u, v, t_{i}\right\rangle$ to decrease the $\nu_{i}$ 's until $\nu_{i}=1$ or $\nu_{i}=0$ for $i=1, \ldots, n$.

Step 2. Blow up ideals of the form $\left\langle u, v, t_{i}, t_{j}\right\rangle, i \neq j$, to decrease the number of variables occurring in $g\left(t_{1}, \ldots, t_{n}\right)$.

Denote one of the morphisms in Step 1 or Step 2 by $h: X \rightarrow Y$ and the exceptional locus by $E$. It is easy to see $H^{q}\left(E,{ }^{\theta_{E}}(p)\right)=0$ for $q>0$ and $p \geqslant 0$. Using decreasing induction on $p$ and the exact sequence

$$
0 \rightarrow \hat{\vartheta}_{X}(p+1) \rightarrow \hat{\vartheta}_{X}(p) \rightarrow \mathcal{Q}_{E}(p) \rightarrow 0,
$$

we obtain $\mathbf{R} h_{*} \theta_{X} \cong \theta_{Y}$.

Remarks. (i) The type of singularities discussed in Proposition 2 occurs in stable curves over a regular base scheme. Using flat base change, it follows that a stable curve over a scheme with rational singularities has rational singularities itself. (ii) One example: Surprisingly $u^{2}-v^{2}-t_{1}^{3}-t_{2}^{7}$ defines a rational singularity while $u^{2}-t_{1}^{3}-t_{2}^{7}$ does not.

\section{REFERENCES}

1. A. B. Altman, R. T. Hoobler and S. L. Kleiman, $A$ note on the base change map for cohomology, Compositio Math. 27 (1973), 25-38. MR 49 \#2740.

2. H. Hironaka, Resolution of singularities of an algebraic variety over a field of characteristic zero. I, II, Ann. of Math. (2) 79 (1964), 109-326. MR 33 \#333.

3. G. Kempf, F. Knudsen, D. Mumford and B. Saint-Donat, Toroidal embeddings. I, Lecture Notes in Math., vol. 339, Springer-Verlag, Berlin and New York, 1973. MR 49 \#299.

4. H. Popp, Fundamentalgruppen algebraischer Mannigfaltigkeiten, Lecture Notes in Math., vol. 176, Springer-Verlag, Berlin and New York, 1970. MR 43 \#3273.

5. E. Viehweg, Canonical divisors and the additivity of the Kodaira dimension for morphisms of relative dimension one, Compositio Math. (to appear).

Institut für Mathematik Und Informatik, Universität Mannheim, D-68 Mannheim, Federal Republic of Germany 